\title{
Robust Transient Stabilization of a Synchronous Generator in an Uncertain Power Network with Transfer Conductances
}

\author{
G. Damm, F. Lamnabhi-Lagarrigue and C.M. Verrelli
}

\begin{abstract}
The robust transient stability problem of a synchronous generator in an uncertain power network with transfer conductances is addressed. A robust adaptive nonlinear feedback control algorithm is designed on the basis of a third order model of the synchronous machine: only two system parameters (synchronous machine damping and inertia constants) along with upper and lower bounds on the remaining uncertain ones are supposed to be known. The conditions to be satisfied by the remote network dynamics for guaranteeing $\mathcal{L}_{2}$ and $\mathcal{L}_{\infty}$ robustness and asymptotic relative speed regulation to zero are weaker than those required by the single machineinfinite bus approximation.
\end{abstract}

\section{INTRODUCTION}

Power networks are among the most complex large-scale, interconnected nonlinear systems ([3], [12]). Mechanical and electrical perturbations such as load shedding, generation tripping or short circuits may force one or more generators to go out of step and to be disconnected from the network. The transient stabilization problem consists in the design of a suitable excitation feedback control keeping each generator close to the synchronous speed when mechanical and electrical perturbations occur (voltage regulation is not considered at this stage). On the basis of linear approximations around operating conditions, decentralized linear controllers were first designed, which however may not be able to guarantee the power grid stability (see [10]) and to handle the severe disturbances and contingencies typically occurring in power networks. In the recent years, several nonlinear algorithms have been proposed for power systems control. For a particular power systems structure $\mathcal{M}$ consisting of a group of generators tied together by a strong network of transmission lines and linked to a single generator by a comparatively weak set of tie lines, the single machine-infinite bus approximation, which models all the remaining network as a fixed voltage source and an impedance, is advantageous in the nonlinear control design: transient stabilization can be achieved even without requiring the knowledge of critical parameters ([5], [6], [7], [10], [14], [15], [20], [22], [26], [27]). In particular, $\mathcal{L}_{2}$ and $\mathcal{L}_{\infty}$ robustness and asymptotic relative speed regulation to zero are achieved in [15] despite

The research was supported by the Italian Ministry of University and Research (PRIN 06) and through a European Community Marie Curie Fellowship (in the framework of the CTS), c.n. HPMT-CT-2001-00278.

G. Damm is with the Laboratoire IBISC-CNRS, Universite d'Evry Val d'Essonne, 40 rue du Pelvoux 91020 Evry Cedex, France, gilney.dammeibisc.fr. F. Lamnabhi-Lagarrigue is with the Laboratoire des Signaux et Systèmes, L2S-CNRS, Plateau de Moulon, Gif sur Yvette 91192, France, lamnabhi@lss.supelec.fr. C.M. Verrelli is with the Electronic Engineering Department of "Tor Vergata" University, Via del Politecnico 1, 00133 Rome, Italy, verrelli@ing.uniroma2.it. uncertainties in all system parameters.

In this paper, we address the transient stabilization problem for the particular power systems structure $\mathcal{M}$ above considered. The single machine-infinite bus model is however not used so that remote network dynamics effects are taken into account. Following the theoretical developments in [16], [17], [21], a robust adaptive nonlinear feedback control is designed which does not assume the knowledge of the system parameters excepting for the machine damping and inertia constants. On the basis of upper and lower bounds on the uncertain model parameters, $\mathcal{L}_{2}$ and $\mathcal{L}_{\infty}$ robustness and asymptotic relative speed regulation to zero are guaranteed under a set of assumptions on the network dynamics which are weaker than those required by the single machineinfinite bus approximation, while the controller parameters are adapted using the robust adaptive techniques in [16]. Simulation results with reference to a 3-machine, 9-buses power network illustrate the effectiveness of the adopted approach, showing that the proposed robust nonlinear excitation control prevents each network machine from going out of step in the presence of electrical parameter perturbations and unmodelled dynamics and improves the performance with respect to the controller in [15] based on the single machine-infinite bus approximation. The presented approach in conjuction with the special structure of power systems considered here allows us, without assuming the availability of the machine internal voltage measurement, to solve the robust transient stabilization problem of a synchronous generator in a power network in the presence of transfer conductances and uncertainties in the most of system parameters, which is typically a difficult result to obtain for general structures of power systems (see for instance [9], [11], [13], [18], [20], [23], [24], [25], [28], [29], [30]).

\section{SYSTEM DYNAMIC MODEL}

A power system consisting of $n$ generators interconnected through a transmission network is described by the $3 n$-order nonlinear model in [19] $[1 \leq i \leq n]$

$$
\begin{aligned}
\dot{\delta}_{i} & =\omega_{i} \\
\dot{\omega}_{i} & =-\frac{D_{i}}{2 H_{i}} \omega_{i}+\frac{\omega_{0}}{2 H_{i}} P_{m i}-\frac{\omega_{0}}{2 H_{i}} P_{e i} \\
\dot{E}_{q i}^{\prime} & =\frac{k_{c i}}{T_{d 0 i}^{\prime}} u_{f i}-\frac{E_{q i}^{\prime}}{T_{d 0 i}^{\prime}}-\frac{\left(x_{d i}-x_{d i}^{\prime}\right)}{T_{d 0 i}^{\prime}} I_{d i}
\end{aligned}
$$

in which the first two equations represent the $i$-th generator mechanical dynamics involving the the power angle $\delta_{i}(\mathrm{rad})$, the relative angular speed $\omega_{i}(\mathrm{rad} / \mathrm{s})$, the active electrical power $P_{e i}$ (p.u.), the mechanical input power $P_{m i}$ (p.u.), the 
synchronous speed $\omega_{0}(\mathrm{rad} / \mathrm{s})$, the damping constant $D_{i}$ (p.u.) and the inertia constant $H_{i}(\mathrm{~s})$, while the third equation constitutes the $i$-th generator electrical dynamics involving the transient EMF $E_{q i}^{\prime}$ (p.u.) in the quadrature axis, the input $u_{f i}$ (p.u.) to the thyristor amplifier, the gain $k_{c i}$ of the excitation amplifier, the direct axis transient open circuit time constant $T_{d 0 i}^{\prime}(\mathrm{s})$, the direct axis reactance $x_{d i}$ (p.u.) and the direct axis transient reactance $x_{d i}^{\prime}$ (p.u.). The $i$-th generator electrical equations are

$$
\begin{aligned}
P_{e i}= & E_{q i}^{\prime 2} G_{i i}+E_{q i}^{\prime} \sum_{j=1, j \neq i}^{n}\left[E_{q j}^{\prime} G_{i j} \cos \left(\delta_{i j}\right)\right. \\
& \left.+E_{q j}^{\prime} B_{i j} \sin \left(\delta_{i j}\right)\right] \\
Q_{e i}= & -E_{q i}^{\prime 2} B_{i i}+E_{q i}^{\prime} \sum_{j=1, j \neq i}^{n}\left[E_{q j}^{\prime} G_{i j} \sin \left(\delta_{i j}\right)\right. \\
& \left.-E_{q j}^{\prime} B_{i j} \cos \left(\delta_{i j}\right)\right]-x_{d i}^{\prime}\left(I_{d i}^{2}+I_{q i}^{2}\right) \\
I_{d i}= & -E_{q i}^{\prime} B_{i i}+\sum_{j=1, j \neq i}^{n}\left[E_{q j}^{\prime} G_{i j} \sin \left(\delta_{i j}\right)\right. \\
& \left.-E_{q j}^{\prime} B_{i j} \cos \left(\delta_{i j}\right)\right] \\
I_{q i}= & E_{q i}^{\prime} G_{i i}+\sum_{j=1, j \neq i}^{n}\left[E_{q j}^{\prime} G_{i j} \cos \left(\delta_{i j}\right)\right. \\
& \left.+E_{q j}^{\prime} B_{i j} \sin \left(\delta_{i j}\right)\right] \\
\delta_{i j}= & \delta_{i}-\delta_{j} \\
V_{t i}= & \sqrt{\left(x_{d i}^{\prime} I_{q i}\right)^{2}+\left(E_{q i}^{\prime}-x_{d i}^{\prime} I_{d i}\right)^{2}}
\end{aligned}
$$

in which (for the $i$-th generator): $Q_{e i}$ (p.u.) is the reactive electrical power, $I_{d i}$ (p.u.) is the direct axis current, $I_{q i}$ (p.u.) is the quadrature axis current, $V_{t i}$ (p.u.) is the terminal voltage, $G_{i j}$ (p.u.) and $B_{i j}$ (p.u.) are the $i$-th row and the $j$ th column element of nodal conductance and susceptance matrices, respectively, at the internal nodes after eliminating all physical buses, which depend on $x_{d i}^{\prime}$ (p.u.), on the transformer reactance $x_{T i}$ (p.u.), on the loads and on the transmission line reactance $x_{i j}$ (p.u.) between the $i$-th generator and the $j$-th generator. The nodal conductance and susceptance matrices represent the full power network of transmission lines and loads connecting the power generators.

In this paper, we study a particular structure of power systems, i.e., a group $G_{M}$ of $n-1$ generators, tied together by a strong network of transmission lines, which is linked to a generator $g_{m}$ (in the following referred to as $r$-th generator) by a comparatively weak set of tie lines. Accordingly, we explicitly compute $\dot{P}_{e r}\left[G_{r j}\right.$ and $B_{r j}, 1 \leq j \leq n$, are assumed to be constant], so that the $r$-th generator third order dynamic model can be written as $\left[I_{q r}>c_{I r}>0(\text { see }[20])^{1}\right]$

$$
\dot{\delta}_{r}=\omega_{r}
$$

${ }^{1}$ We assume that there exist bounded connected open sets $\mathcal{D}_{\delta}$ and $\mathcal{D}_{p}$ such that $E_{q r}^{\prime}(0)>0, \delta_{r}(t) \in \mathcal{D}_{\delta}$ and $P_{e r}(t) \in \mathcal{D}_{p}$ imply $I_{q r}(t)>$ $c_{\text {Ir }}>0$, which describes the whole practical operating region of the generator.

$$
\begin{aligned}
\dot{\omega}_{r}= & -\frac{D_{r}}{2 H_{r}} \omega_{r}+\frac{\omega_{0}}{2 H_{r}} P_{m r}-\frac{\omega_{0}}{2 H_{r}} P_{e r} \\
\dot{P}_{e r}= & -\theta_{1 r} P_{e r}-\theta_{2 r} I_{d r} I_{q r}-\theta_{3 r} \frac{I_{d r} P_{e r}}{I_{q r}}-\theta_{4 r} \frac{P_{e r}^{2}}{I_{q r}^{2}} \\
& +\left[\frac{\theta_{5 r} I_{q r}^{2}+\theta_{6 r} P_{e r}}{I_{q r}}\right] u_{f r}-\left(Q_{e r}+B_{r r} \frac{P_{e r}^{2}}{I_{q r}^{2}}\right. \\
& \left.+x_{d r}^{\prime}\left(I_{d r}^{2}+I_{q r}^{2}\right)\right) \omega_{r}+\frac{P_{e r}}{I_{q r}} R_{r}
\end{aligned}
$$

in which $\delta_{r}, \omega_{r}, P_{e r}$ are the state variables, $u_{f r}$ is the control input, $\theta_{1 r}=\frac{1}{T_{d 0 r}^{\prime}}, \theta_{2 r}=\frac{\left(x_{d r}-x_{d r}^{\prime}\right)}{T_{d 0 r}^{\prime}}, \theta_{3 r}=\frac{G_{r r}\left(x_{d r}-x_{d r}^{\prime}\right)}{T_{d 0 r}^{\prime}}$, $\theta_{4 r}=\frac{G_{r r}}{T_{d 0 r}^{\prime}}, \theta_{5 r}=\frac{k_{c r}}{T_{d 0 r}^{\prime}}, \theta_{6 r}=\frac{G_{r r} k_{c r}}{T_{d 0 r}^{\prime}}$ and the term

$$
\begin{aligned}
R_{r}= & \sum_{j=1, j \neq r}^{n} \dot{\tilde{E}}_{q j}^{\prime}\left[G_{r j} \cos \left(\delta_{r j}\right)+B_{r j} \sin \left(\delta_{r j}\right)\right] \\
& +\sum_{j=1, j \neq r}^{n} \omega_{j}\left[E_{q j}^{\prime} G_{r j} \sin \left(\delta_{r j}\right)-E_{q j}^{\prime} B_{r j} \cos \left(\delta_{r j}\right)\right]
\end{aligned}
$$

represents the effect of the network remote dynamics (the group $G_{M}$ of generators) on the $r$-th generator, where $\tilde{E}_{q j}^{\prime}=$ $E_{q j}^{\prime}-E_{q j 0}^{\prime}$, with $E_{q j 0}^{\prime}$ the pre-fault equilibrium value for $E_{q j}^{\prime}$. In the practice, the exact values of the model parameters are hard to obtain, and in particular $P_{m}, G_{r r}, B_{r r}$ are lumped parameters which account for unmodelled dynamics such as turbine and load dynamics. Those parameters may undergo sudden on line variations due to mechanical and electrical perturbations and faults. In the following, we will suppose the parameters $\omega_{0}, D_{r}, H_{r}$ to be known and will assume that: the uncertain piecewise continuous parameters $T_{d 0 r}^{\prime}(t), x_{d r}(t), x_{d r}^{\prime}(t), k_{c r}(t)$ and the uncertain constant parameter $G_{r r}$ are within the corresponding known positive bounds $\left(T_{d 0 r m}^{\prime}, T_{d 0 r M}^{\prime}\right),\left(x_{d r m}, x_{d r M}\right),\left(x_{d r m}^{\prime}\right.$, $\left.x_{d r M}^{\prime}\right),\left(k_{c r m}, k_{c r M}\right),\left(G_{r r m}, G_{r r M}\right)$; the uncertain constant parameter $B_{r r}$ is within the corresponding known bounds $\left(B_{r r m}, B_{r r M}\right)$; the mechanical input power $P_{m r}(t) \in \mathcal{D}_{p}$ is a class $\mathcal{C}^{1}$ function satisfying: $P_{m r M} \geq P_{m r}(t) \geq P_{m r m}$ and $\left|\dot{P}_{m r}(t)\right| \leq \dot{P}_{M r}$, with $P_{m r m}, P_{m r M}, \dot{P}_{M r}$ known positive reals. Physical considerations concerning transmission lines, loads and mechanical turbines make the above assumptions reasonable in the transient stabilization problem of power networks.

\section{PRoblem StATEMENT}

A suitable control problem formulation is introduced in this section, which will allow us to solve the transient stabilization problem for the synchronous generator $g_{m}$ by quantitatively characterizing the robustness with respect to both permanent and vanishing model parameter perturbations. Denote by $\delta_{r s} \in \mathcal{D}_{\delta}$ the pre-fault constant value for the power angle $\delta_{r}$ and let $\theta_{i r m}, \theta_{i r M}$ be the known positive bounds on the uncertain parameters $\theta_{i r}(t), 1 \leq i \leq 6$, with $\hat{P}_{m r}(t)$ a suitable estimate of the uncertain mechanical power $P_{m r}$. The transient stabilization problem addressed in this paper is rigorously formulated as follows.

Definition 1 (Transient Stabilizing Control): Assume that for any bounded piecewise continuous real-valued function 
$u_{f r}(\cdot)$ defined on $\mathbb{R}_{0}^{+}$and for all $t \geq 0$ : i) for each $j \neq r$, $1 \leq j \leq n$, the $j$-th generator power angle $\delta_{j}(t)$, the relative angular speed $\omega_{j}(t)$ and the quadrature axis transient EMF $E_{q j}^{\prime}(t)$ are piecewise differentiable functions of time $t$ and boundedness of the $r$-th generator variables $\delta_{r}(t), \omega_{r}(t)$, $P_{e r}(t)$ implies boundedness of $E_{q j}^{\prime}(t)$; ii) there exist $\mu_{r}$, $\nu_{r}, \rho_{r}$ (unknown) non-negative reals, $\bar{\varphi}_{\mu r}(\cdot), \bar{\varphi}_{\nu r}(\cdot), \bar{\varphi}_{\rho r}(\cdot)$ known $\mathcal{K}_{\infty}$ functions and $g_{r}(t)$ (unknown) bounded nonnegative real-valued function of time $t$ such that

$$
\begin{aligned}
\left|R_{r}(t)\right| \leq & \sup _{0 \leq \tau \leq t}\left\{g_{r}(\tau)\right\}+\mu_{r} \bar{\varphi}_{\mu r}\left(\max _{0 \leq \tau \leq t}\left\{\left|\delta_{r}(\tau)-\delta_{r s}\right|\right\}\right) \\
& +\nu_{r} \bar{\varphi}_{\nu r}\left(\max _{0 \leq \tau \leq t}\left\{\left|\omega_{r}(\tau)\right|\right\}\right) \\
& +\rho_{r} \bar{\varphi}_{\rho r}\left(\max _{0 \leq \tau \leq t}\left\{\left|P_{e r}(\tau)-P_{m r}(\tau)\right|\right\}\right) .
\end{aligned}
$$

Define $\left[\varepsilon_{j r}, \varepsilon_{x r}, \varepsilon_{B r}(1 \leq j \leq 6)\right.$ are positive reals]:

$$
\begin{aligned}
y_{r}(t)= & {\left[\delta_{r}(t)-\delta_{r s}, \omega_{r}(t), P_{e r}(t)-P_{m r}(t)\right]^{T} } \\
\xi_{r}(t)= & {\left[y_{r}(t)^{T}, P_{m r}(t)-\hat{P}_{m r}(t)\right]^{T} } \\
w_{d r}(t)= & {\left[\dot{P}_{m r}(t), \theta_{1 r M}-\theta_{1 r m}+\varepsilon_{1 r}, \theta_{2 r M}-\theta_{2 r m}\right.} \\
& +\varepsilon_{2 r}, \theta_{3 r M}-\theta_{3 r m}+\varepsilon_{3 r}, \theta_{4 r M}-\theta_{4 r m}+\varepsilon_{4 r}, \\
& \max \left\{\theta_{5 r M}-\theta_{5 r m}+\varepsilon_{5 r}, \theta_{6 r M}-\theta_{6 r m}+\varepsilon_{6 r}\right\}, \\
& x_{d r M}^{\prime}-x_{d r m}^{\prime}+\varepsilon_{x r}, B_{r r M}-B_{r r m}+\varepsilon_{B r}, \\
& \left.\sup _{0 \leq \tau \leq t}\left\{g_{r}(\tau)\right\}, \mu_{r}, \nu_{r}, \rho_{r}\right]^{T} .
\end{aligned}
$$

A bounded control law $u_{f r}$ is called a transient stabilizing control for the $r$-th generator if it guarantees the closed loop system to satisfy the following: (S1) $\mathcal{L}_{\infty}$ disturbance attenuation property, i.e.

$$
\left\|y_{r}(t)\right\|^{2} \leq h_{1 r}\left(\xi_{r}(0)\right) e^{-c_{r} t}+\frac{1}{k_{r}} \gamma_{1 r}\left(\left\|w_{d r}(\cdot)\right\|_{\infty}\right)
$$

holds for all $t \geq 0$, where $h_{1 r}\left(\xi_{r}(0)\right) \geq 0, c_{r}>0$ and $\gamma_{1 r}(r)$ is a class $\mathcal{K}_{\infty}$ function; (S2) $\mathcal{L}_{2}$ disturbance attenuation property, i.e.

$\int_{0}^{T}\left\|y_{r}(\tau)\right\|^{2} d \tau \leq h_{2 r}\left(\xi_{r}(0)\right)+\frac{1}{k_{r}} \int_{0}^{T} \gamma_{2 r}\left(\left\|w_{d r}(\tau)\right\|\right) d \tau$

holds for any given $T>0$, where $h_{2 r}\left(\xi_{r}(0)\right) \geq 0$ and $\gamma_{2 r}(r)$ is a class $\mathcal{K}_{\infty}$ function.

Definition 2 (Transient Adaptive Stabilizing Control): Assume that, in addition to assumptions i)-ii), there exist $t_{0}, M_{R r}, \gamma_{\delta r}, \gamma_{\omega r}$ and $\gamma_{p r}$ non-negative reals such that: iii) the uncertain machine parameters $P_{m r}(t), T_{d 0 r}^{\prime}(t), x_{d r}(t)$, $x_{d r}^{\prime}(t), k_{c r}(t)$ are constant for all $\left.t \geq t_{0} ; i v\right)$ the following inequality:

$$
\begin{aligned}
\lim _{t \rightarrow \infty} \int_{t_{0}}^{t} R_{r}^{2}(\tau) d \tau \leq & M_{R r}+\gamma_{\delta r} \lim _{t \rightarrow \infty} \int_{t_{0}}^{t}\left[\delta_{r}(\tau)-\delta_{r s}\right]^{2} d \tau \\
& +\gamma_{\omega r} \lim _{t \rightarrow \infty} \int_{t_{0}}^{t}\left[\omega_{r}(\tau)\right]^{2} d \tau \\
& +\gamma_{p r} \lim _{t \rightarrow \infty} \int_{t_{0}}^{t}\left[P_{e r}(\tau)-P_{m r}(\tau)\right]^{2} d \tau
\end{aligned}
$$

holds. A transient stabilizing control $u_{f r}$ is called a transient adaptive stabilizing control for the $r$-th generator if it guarantees, the additional property: (S3) asymptotic regulation, i.e.

$$
\lim _{t \rightarrow \infty}\left\|\left[\delta_{r}(t)-\delta_{r s}, \omega_{r}(t), P_{e r}(t)-P_{m r}\right]\right\|=0 .
$$

\section{NONLINEAR DESIGN AND STABILITY ANALYSIS}

By virtue of techniques similar to those used in [7], we design, in this section, a transient adaptive stabilizing control for the $r$-th generator according to Definitions 1 and 2 . Define the power angle regulation and relative angular speed tracking errors: $\tilde{\delta}_{r}=\delta_{r}-\delta_{r s}, \tilde{\omega}_{r}=\omega_{r}-\omega_{r}^{*}$, with $\delta_{r s}$ being the power angle constant reference value and $\omega_{r}^{*}$ being the relative angular speed time-varying reference signal $\left[k_{\delta r}\right.$ is a positive control parameter]

$$
\omega_{r}^{*}=-\frac{5}{4} k_{\delta r} \tilde{\delta}_{r} .
$$

Design the active electrical power time-varying reference signal $P_{e r}^{*}$ as $\left[k_{\omega r}, k_{\omega p r}\right.$ are positive control parameters]

$$
\begin{aligned}
P_{e r}^{*}= & \frac{2 H_{r}}{\omega_{0}}\left[\frac{5}{4} k_{\omega r} \tilde{\omega}_{r}+\frac{5}{4} k_{\delta r} \omega_{r}-\frac{D_{r}}{2 H_{r}} \omega_{r}^{*}+\tilde{\delta}_{r}\right. \\
& \left.+\frac{1}{k_{\omega p r}} \tilde{\omega}_{r}\right]+\hat{P}_{m r}
\end{aligned}
$$

which relies on the estimate $\hat{P}_{m r}$ of the uncertain mechanical power $P_{m r}$ satisfying the estimation law $\left[k_{p e r}, k_{r}\right.$ are positive control parameters]

$$
\begin{aligned}
\hat{P}_{m r}= & \phi_{r}+\frac{2 H_{r}}{\omega_{0}}\left(\frac{5}{4} k_{p e r}+\frac{k_{r}}{4}+\frac{1}{k_{r}}+\frac{\omega_{0}^{2}}{16 H_{r}^{2}} k_{\omega p r}\right) \omega_{r} \\
\dot{\phi}_{r}= & \left(\frac{5}{4} k_{p e r}+\frac{k_{r}}{4}+\frac{1}{k_{r}}+\frac{\omega_{0}^{2}}{16 H_{r}^{2}} k_{\omega p r}\right)\left[-\phi_{r}\right. \\
& +\frac{D_{r}}{\omega_{0}} \omega_{r}+P_{e r}-\frac{2 H_{r}}{\omega_{0}}\left(\frac{5}{4} k_{p e r}+\frac{k_{r}}{4}+\frac{1}{k_{r}}\right. \\
& \left.\left.+\frac{\omega_{0}^{2}}{16 H_{r}^{2}} k_{\omega p r}\right) \omega_{r}\right] \\
P_{m r m} \leq & \hat{P}_{m r}(0) \leq P_{m r M}
\end{aligned}
$$

and introduce the active electrical power tracking error: $\tilde{P}_{e r}=P_{e r}-P_{e r}^{*}$. Design the robust control law $u_{f r}$ with the stabilizing and robustifying terms $v_{r}, \varpi_{r}$ as $\left[k_{p r}, k_{R r}\right.$ are positive control parameters]

$$
\begin{aligned}
u_{f r}= & \frac{I_{q r}}{\left(\hat{\theta}_{5 r} I_{q r}^{2}+\hat{\theta}_{6 r} P_{e r}\right)} v_{r} \\
& -\frac{k_{r} I_{q r}\left(2 I_{q r}^{4}+2 P_{e r}^{2}\right) v_{r}^{2} \tilde{P}_{e r}}{4\left(\hat{\theta}_{5 r} I_{q r}^{2}+\hat{\theta}_{6 r} P_{e r}\right)^{2}\left(\theta_{5 r m} I_{q r}^{2}+\theta_{6 r m} P_{e r}\right)} \\
v_{r}= & -\frac{5}{4} k_{p r} \tilde{P}_{e r}+\frac{\omega_{0}}{2 H_{r}} \tilde{\omega}_{r}+\frac{5 D_{r} k_{\delta r}}{4 \omega_{0}} \omega_{r} \\
& +\frac{2 H_{r}}{\omega_{0}} \omega_{r}+\hat{\theta}_{1 r} P_{e r}+\hat{\theta}_{2 r} I_{d r} I_{q r}+\hat{\theta}_{3 r} \frac{I_{d r} P_{e r}}{I_{q r}}
\end{aligned}
$$




$$
\begin{aligned}
& +\hat{\theta}_{4 r} \frac{P_{e r}^{2}}{I_{q r}^{2}}+\hat{B}_{r r} \frac{P_{e r}^{2} \omega_{r}}{I_{q r}^{2}}+\hat{x}_{d r}^{\prime}\left(I_{d r}^{2}+I_{q r}^{2}\right) \omega_{r} \\
& +Q_{e r} \omega_{r}-\frac{5 k_{\delta r}}{4 \omega_{0}}\left[D_{r} \omega_{r}-\omega_{0}\left(\hat{P}_{m r}-P_{e r}\right)\right] \\
& -\frac{2 H_{r}}{\omega_{0}}\left(\frac{5}{4} k_{\omega r}+\frac{1}{k_{\omega p r}}\right)\left[\left(\frac{5}{4} k_{\omega r}+\frac{D_{r}}{2 H_{r}}\right) \tilde{\omega}_{r}+\tilde{\delta}_{r}\right. \\
& \left.+\frac{\omega_{0}}{2 H_{r}} \tilde{P}_{e r}+\frac{1}{k_{\omega p r}} \tilde{\omega}_{r}\right]+\varpi_{r} \\
\varpi_{r}= & -\frac{k_{R r}}{4} \frac{P_{e r}^{2}}{I_{q r}^{2}} \tilde{P}_{e r}-\frac{k_{r}}{4} \tilde{P}_{e r}\left[P_{e r}^{2}+I_{d r}^{2} I_{q r}^{2}+\frac{I_{d r}^{2} P_{e r}^{2}}{I_{q r}^{2}}\right. \\
& \left.+\frac{P_{e r}^{4}}{I_{q r}^{4}}\left(1+\omega_{r}^{2}\right)+\omega_{r}^{2}\left(I_{d r}^{2}+I_{q r}^{2}\right)^{2}\right]-\frac{k_{r}}{4}\left(\frac{5}{4} k_{\omega r}\right. \\
& +\frac{5}{4} k_{\delta r}+\frac{5}{4} k_{p e r}+\frac{k_{r}}{4}+\frac{1}{k_{r}}+\frac{\omega_{0}^{2}}{16 H_{r}^{2}} k_{\omega p r} \\
& \left.+\frac{1}{k_{\omega p r}}\right)^{2} \tilde{P}_{e r}-\frac{k_{r}}{4} \frac{P_{e r}^{2}}{I_{q r}^{2}} \tilde{P}_{e r}\left[\bar{\varphi}_{\mu r}^{2}\left(\max _{0 \leq \tau \leq t}\left\{\tilde{\delta}_{r}(\tau)\right\}\right)\right. \\
& +\bar{\varphi}_{\nu r}^{2}\left(\max _{0 \leq \tau \leq t}\left\{\tilde{\omega}_{r}(\tau)\right\}+\frac{5}{4} k_{\delta r} \max _{0 \leq \tau \leq t}\left\{\tilde{\delta}_{r}(\tau)\right\}\right) \\
& +\bar{\varphi}_{\rho r}^{2}\left(\max _{0 \leq \tau \leq t}\left\{\tilde{P}_{e r}(\tau)\right\}+P_{m r M}-P_{m r m}+\frac{\dot{P}_{M r}}{k_{p e r}}\right. \\
& +\frac{2 H_{r}}{\omega_{0}}\left(\frac{5}{4} k_{\omega r}+\frac{1}{k_{\omega p r}}+\frac{5}{4} k_{\delta r}\right) \max _{0 \leq \tau \leq t}\left\{\tilde{\omega}_{r}(\tau)\right\} \\
& \left.+\left[\frac{2 H_{r}}{\omega_{0}}\left(1+\frac{25}{16} k_{\delta r}^{2}\right)+\frac{5 D_{r} k_{\delta r}}{4 \omega_{0}}\right] \max _{0 \leq \tau \leq t}\left\{\tilde{\delta}_{r}(\tau)\right\}\right) \\
& +1]
\end{aligned}
$$

whose the time derivative of function $\mathcal{V}_{r}$ along the trajectories of the error system satisfies

$$
\begin{aligned}
\dot{\mathcal{V}}_{r} \leq & -\frac{5}{4}\left(k_{\delta r} \tilde{\delta}_{r}^{2}+k_{\omega r} \tilde{\omega}_{r}^{2}+k_{p r} \tilde{P}_{e r}^{2}+k_{p e r} \tilde{P}_{m r}^{2}\right) \\
& +\frac{1}{k_{r}}\left[\dot{P}_{m r}^{2}+\tilde{\theta}_{1 r}^{2}+\tilde{\theta}_{2 r}^{2}+\tilde{\theta}_{3 r}^{2}+\tilde{\theta}_{4 r}^{2}\right. \\
& +\max \left\{\tilde{\theta}_{5 r}^{2}, \tilde{\theta}_{6 r}^{2}\right\}+\tilde{x}_{d r}^{2}+\tilde{B}_{i r}^{2} \\
& \left.+\sup _{0 \leq \tau \leq t}\left\{g_{r}(\tau)\right\}^{2}+\mu_{r}^{2}+\nu_{r}^{2}+\rho_{r}^{2}\right]
\end{aligned}
$$

we can establish [recall property 1 . of the projection algorithm] that the robust adaptive nonlinear feedback control algorithm (3)-(7) is a transient stabilizing control for the $r$ th generator. Suppose that, in addition to assumptions i)-ii), assumptions iii)-iv) hold and consider the quadratic function $\left[\beta_{j r}, \beta_{x r}, \beta_{B r}(1 \leq j \leq 6)\right.$ are positive reals]

$$
\mathcal{W}_{r}=\mathcal{V}_{r}+\frac{1}{2} \sum_{j=1}^{6} \beta_{j r} \tilde{\theta}_{j r}^{2}+\frac{1}{2} \beta_{x r} \tilde{x}_{d r}^{\prime 2}+\frac{1}{2} \beta_{B r} \tilde{B}_{i r}^{2}
$$

whose time derivative along the trajectories of the error system, for all $t \geq t_{0}$, satisfies [recall property 4 . of the projection algorithm]

$$
\begin{aligned}
\dot{\mathcal{W}}_{r} \leq & -\frac{5}{4} k_{\delta r} \tilde{\delta}_{r}^{2}-\frac{5}{4} k_{\omega r} \tilde{\omega}_{r}^{2}-\frac{5}{4} k_{p r} \tilde{P}_{e r}^{2} \\
& -\frac{5}{4} k_{p e r} \tilde{P}_{m r}^{2}+\frac{R_{r}(t)^{2}}{k_{R r}}
\end{aligned}
$$

which rely on the estimates $\hat{\theta}_{j r}, \hat{x}_{d r}^{\prime}, \hat{B}_{r r}$ of the uncertain parameters $\theta_{j r}, x_{d r}^{\prime}, B_{r r}$ whose estimation laws $(1 \leq j \leq 6)$ [functions $\xi_{\theta_{j}}(t), \xi_{x}(t), \xi_{b}(t)$ are yet to be chosen]

$$
\begin{aligned}
\dot{\hat{\theta}}_{j r} & =\operatorname{Proj}\left[\xi_{\theta_{j}}(t), \hat{\theta}_{j r}, \theta_{j r m}, \theta_{j r M}, \varepsilon_{j r}\right] \\
\theta_{j r m} & \leq \hat{\theta}_{j r}(0) \leq \theta_{j r M} \\
\dot{\hat{x}}_{d r}^{\prime} & =\operatorname{Proj}\left[\xi_{x}(t), \hat{x}_{d r}^{\prime}, x_{d r m}^{\prime}, x_{d r M}^{\prime}, \varepsilon_{x r}\right] \\
x_{d r m}^{\prime} & \leq \hat{x}_{d r}^{\prime}(0) \leq x_{d r M}^{\prime} \\
\dot{\hat{B}}_{r r} & =\operatorname{Proj}\left[\xi_{b}(t), \hat{B}_{r r}, B_{r r m}, B_{r r M}, \varepsilon_{B r}\right] \\
B_{r r m} & \leq \hat{B}_{r r}(0) \leq B_{r r M}
\end{aligned}
$$

are designed by using the projection algorithm $\operatorname{Proj}\left[\zeta, \hat{z}_{r}, z_{r m}, z_{r M}, \varepsilon_{z r}\right]$ in [15] $\left[\left(z_{r m}-\varepsilon_{z r}\right)>0\right]$ whose properties are the following [which hold provided that the uncertain constant $z_{r}$ and the initial condition $\hat{z}_{r}(0)$ belong to the compact set $\left.\left[z_{r m}, z_{r M}\right]\right]: 1 . z_{r m}-\varepsilon_{z r} \leq \hat{z}_{r}(t) \leq z_{r M}+\varepsilon_{z r}$, for all $t \geq 0 ; 2$. $\operatorname{Proj}\left[\zeta, \hat{z}_{r}, \cdot, \cdot, \cdot\right]$ is Lipschitz continuous; 3. $\left|\operatorname{Proj}\left[\zeta, \hat{z}_{r}, z_{r m}, z_{r M}, \varepsilon_{z r}\right]\right| \leq|\zeta| ; 4$. $\left(z_{r}-\hat{z}_{r}\right) \operatorname{Proj}\left[\zeta, \hat{z}_{r}, z_{r m}, z_{r M}, \varepsilon_{z r}\right] \geq\left(z_{r}-\hat{z}_{r}\right) \zeta$. Defining the estimation errors $(1 \leq j \leq 6)$ : $\tilde{\theta}_{j r}=\theta_{j r}-\hat{\theta}_{j r}$, $\tilde{x}_{d r}^{\prime}=x_{d r}^{\prime}-\hat{x}_{d r}^{\prime}, \tilde{B}_{r r}=B_{r r}-\hat{B}_{r r}$ and considering the quadratic function

$$
\mathcal{V}_{r}=\frac{1}{2}\left(\tilde{\delta}_{r}^{2}+\tilde{\omega}_{r}^{2}+\tilde{P}_{e r}^{2}+\tilde{P}_{m r}^{2}\right)
$$

provided that the yet to be defined functions $\xi_{\theta_{j}}(t), \xi_{x}(t)$, $\xi_{b}(t)(1 \leq j \leq 6)$ are chosen as

$$
\begin{aligned}
\xi_{\theta_{1}} & =-\frac{P_{e r} \tilde{P}_{e r}}{\beta_{1 r}}, \quad \xi_{\theta_{2}}=-\frac{I_{d r} I_{q r} \tilde{P}_{e r}}{\beta_{2 r}}, \\
\xi_{\theta_{3}} & =-\frac{I_{d r} P_{e r} \tilde{P}_{e r}}{I_{q r} \beta_{3 r}}, \quad \xi_{\theta_{4}}=-\frac{P_{e r}^{2} \tilde{P}_{e r}}{I_{q r}^{2} \beta_{4 r}}, \\
\xi_{\theta_{5}} & =\frac{I_{q r}^{2} v_{r} \tilde{P}_{e r}}{\left(\hat{\theta}_{5 r} I_{q r}^{2}+\hat{\theta}_{6 r} P_{e r}\right) \beta_{5 r}}, \\
\xi_{\theta_{6}} & =\frac{P_{e r} v_{r} \tilde{P}_{e r}}{\left(\hat{\theta}_{5 r} I_{q r}^{2}+\hat{\theta}_{6 r} P_{e r}\right) \beta_{6 r}}, \\
\xi_{x} & =-\frac{\left(I_{d r}^{2}+I_{q r}^{2}\right) \omega_{r} \tilde{P}_{e r}}{\beta_{x r}}, \quad \xi_{b}=-\frac{P_{e r}^{2} \omega_{r} \tilde{P}_{e r}}{\beta_{B r} I_{q r}^{2}} .
\end{aligned}
$$

Since $\tilde{\delta}_{r}, \tilde{\omega}_{r}, \tilde{P}_{e r}, \tilde{P}_{m r}$ are bounded, $\dot{\tilde{\delta}}_{r}, \dot{\tilde{\omega}}_{r}, \dot{\tilde{P}}_{e r}, \dot{\tilde{P}}_{m r}$ are bounded so that $\tilde{\delta}_{r}(t), \tilde{\omega}_{r}(t), \tilde{P}_{e r}(t), \tilde{P}_{m r}(t)$ are uniformly continuous for all $t \geq t_{0}$. On the other hand, by virtue of assumptions iii)-iv), if $k_{R r}>\max \left\{\frac{4 \bar{\gamma}_{\delta r}}{k_{\delta r}}, \frac{4 \bar{\gamma}_{\omega r}}{k_{\omega r}}, \frac{16 \gamma_{p r}}{k_{p r}}, \frac{16 \gamma_{p r}}{k_{p e r}}\right\}$ with

$$
\begin{aligned}
\bar{\gamma}_{\delta r}= & \gamma_{\delta r}+\frac{25}{8} \gamma_{\omega r} k_{\delta r}^{2}+4\left[\frac{2 H_{r}}{\omega_{0}}\left(\frac{25}{16} k_{\delta r}^{2}+1\right)\right. \\
& \left.+\frac{5}{4} \frac{D_{r} k_{\delta r}}{\omega_{0}}\right]^{2} \gamma_{p r} \\
\bar{\gamma}_{\omega r}= & 2 \gamma_{\omega r}+4\left[\frac{2 H_{r}}{\omega_{0}}\left(\frac{5}{4} k_{\omega r}+\frac{5}{4} k_{\delta r}+\frac{1}{k_{\omega p r}}\right)\right]^{2} \gamma_{p r}
\end{aligned}
$$


then, according to (3), (4), (8) and Barbalat's Lemma, we can establish that the robust adaptive nonlinear feedback control algorithm (3)-(7), (9) is a transient adaptive stabilizing control for the $r$-th generator. The result holds for any initial condition (of the $r$-th generator) and positive control parameter $k_{r}$ maintaining, according to $(\mathrm{S} 1), \delta_{r}(t) \in \mathcal{D}_{\delta}$ and $P_{e r}(t) \in \mathcal{D}_{p}$ (guaranteeing $I_{q r}(t)>c_{I r}>0$ ) for all $t \geq 0$. The main result of this paper can be summarized in the following theorem, which somehow ${ }^{2}$ extends the recent theoretical contribution in [15].

Theorem: The robust adaptive nonlinear feedback control algorithm (3)-(7), (9) is:

- a transient stabilizing control for the $r$-th generator;

- a transient adaptive stabilizing control for the $r$-th generator when

$$
k_{R r}>\max \left\{\frac{4 \bar{\gamma}_{\delta r}}{k_{\delta r}}, \frac{4 \bar{\gamma}_{\omega r}}{k_{\omega r}}, \frac{16 \gamma_{p r}}{k_{p r}}, \frac{16 \gamma_{p r}}{k_{p e r}}\right\},
$$

for any initial condition (of the $r$-th generator) and positive control parameter $k_{r}$ maintaining, according to $(S 1), \delta_{r}(t) \in$ $\mathcal{D}_{\delta}$ and $P_{e r}(t) \in \mathcal{D}_{p}$ (guaranteeing $I_{q r}(t)>c_{I r}>0$ ) for all $t \geq 0$.

\section{Simulation RESUlts}

In this section we illustrate the performance and the robustness of the feedback control algorithm (3)-(7), (9) in the presence of unmodelled dynamics: the proposed control is applied to each generator of the popular Western System Coordinating Council (WSCC) 3-machine, 9-bus system reported in [19] and [1] $\left[D_{i}=0,1 \leq i \leq 3\right]$ and described by the two-axis model in [19] from which the model (1) has been derived by neglecting the dynamics of the fast damper-winding $E_{d i}^{\prime}$ and by using the simplification $x_{q i}=x_{d i}^{\prime}$ with $x_{q i}$ (p.u.) being the quadrature axis reactance. The initial conditions for the state variables are computed by systematically solving the load-flow equations of the network and by computing the values of the algebraic variables. For $1 \leq i \leq 3$, the functions $\bar{\varphi}_{\mu i}(\cdot), \bar{\varphi}_{\nu i}(\cdot), \bar{\varphi}_{\rho i}(\cdot)$ are set equal to $\mathbb{I}(\cdot)_{[0, \infty)}[\mathbb{I}(\cdot)$ is the identity function], the control parameters are chosen as $k_{\delta i}=k_{\omega i}=k_{p e i}=k_{\omega p i}=1$, $k_{p i}=720, k_{i}=0.001, k_{R i}=0.1, \beta_{1 i}=\beta_{2 i}=\beta_{3 i}=$ $\beta_{4 i}=\beta_{5 i}=\beta_{6 i}=\beta_{x i}=\beta_{B i}=348000$ while the initial conditions for the parameter estimates are set equal to the corresponding nominal values. In order to avoid division by zero, for $1 \leq i \leq 3, \eta\left(P_{e i}\right)$ and $\eta\left(I_{q i}\right)$ replace $P_{e i}$ and $I_{q i}$ in the control algorithm (3)-(7), (9), respectively, where $\eta(\xi)=\left\{\begin{array}{ll}\xi, & \text { if } \xi \geq 0.05 \\ 0.05, & \text { otherwise. }\end{array}\right.$ The goal of the simulation is to verify the effects of a three-phase fault occurring near bus 7 at the end of line 5-7 at $t=0.001 \mathrm{~s}$, which is cleared at 0.084 $\mathrm{s}$ by opening line 5-7 (circuit breakers reclose at $t=1 \mathrm{~s}$ ). Figure 1-2 show a satisfactory performance of the proposed control even with the physical saturations (which are hit several times according to Fig. 2(b)): despite the considered

\footnotetext{
${ }^{2}$ Here the machine damping and inertia constants are assumed to be known.
}

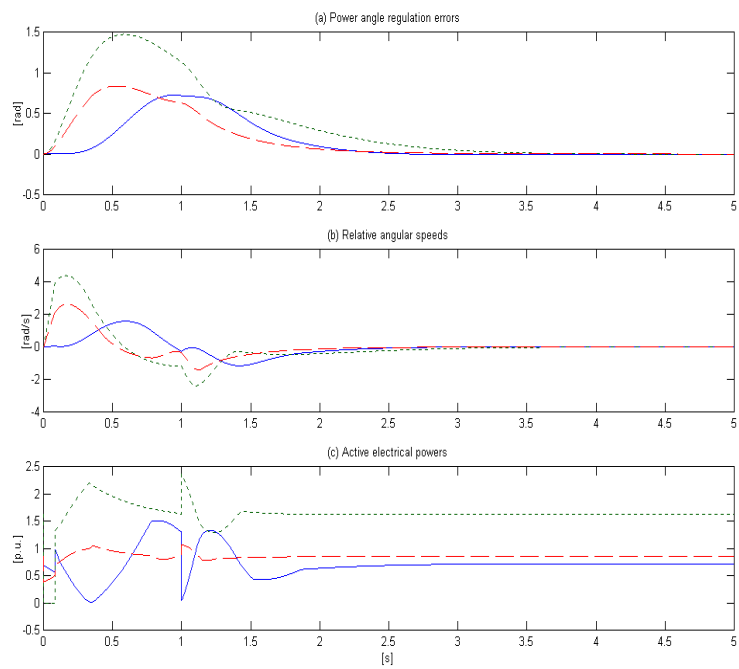

Fig. 1. Proposed control algorithm [Generator 1 (solid), Generator 2 (dot), Generator 3 (dash)]: a) Power angle regulation errors $\delta_{i}-\delta_{i s}(1 \leq i \leq 3)$; b) Relative angular speeds $\omega_{i}(1 \leq i \leq 3)$; c) Active electrical powers $P_{\text {ei }}(1 \leq i \leq 3)$

severe perturbation, synchronous speeds are quickly restored while fast regulation of both power angle and electrical power is guaranteed. For comparison, the same simulation is performed by applying (to each generator of the network) the nonlinear robust adaptive control (with control parameters $k_{\delta}=0.1, k_{\omega}=3, k_{p}=120, k=0.001, \beta_{i}=0.0000001$, $1 \leq i \leq 7$ and initial conditions ${ }^{3} \hat{\theta}_{i}(0)=0 i=1,2,4,5$, $\left.\hat{\theta}_{6}(0)=\frac{u_{f 0} \sin (\delta(0))}{\bar{v}_{2}(0)}, \hat{\theta}_{3}(0)=P_{m}, \hat{\theta}_{7}(0)=1\right)$ designed in [15] on the basis of a (third order) single machine-infinite bus model. As illustrated by the simulation results reported in Fig. 3, neglecting the transient behaviour of the other generators and the interconnections between them may be critical for the control design in [15]: in the presence of the considered perturbation, synchronous speeds can not quickly restored.

\section{CONCLUSIONS}

A robust adaptive nonlinear feedback control (3)-(7), (9) has been designed on the basis of a third order model (1) of a synchronous generator in an uncertain power network with transfer conductances. The proposed controller guarantees the $\mathcal{L}_{2}$ and $\mathcal{L}_{\infty}$ disturbance attenuation and asymptotic regulation properties (S1)-(S3) under assumptions on the network dynamics generalizing those required by the single machine-infinite bus approximation used in [15].

\section{REFERENCES}

[1] P.M. Anderson and A.A. Fouad, Power system control and stability, New York, Wiley-IEEE Press, 2003.

[2] E. Barrera-Cardiel and N. Pastor-Gomez, "Microcontroller-based power-angle instrument for a power systems laboratory", IEEE Power Engineering Society Summer Meeting vol. 2, pp. 1008-1012, 1999.

[3] A.R. Bergen and V. Vittal, Power systems analysis, Upper Saddle River, Prentice Hall, 2000.

${ }^{3}$ We denote by $u_{f 0}$ the nominal input value. 

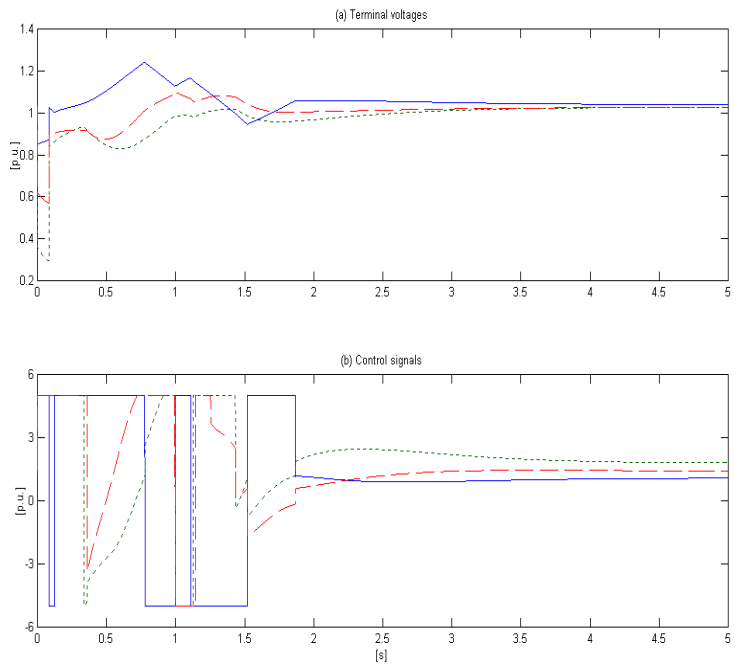

Fig. 2. Proposed control algorithm [Generator 1 (solid), Generator 2 (dot), Generator 3 (dash)]: a) Terminal voltages $V_{t i}(1 \leq i \leq 3)$; b) Control signals $u_{f i}(1 \leq i \leq 3)$.
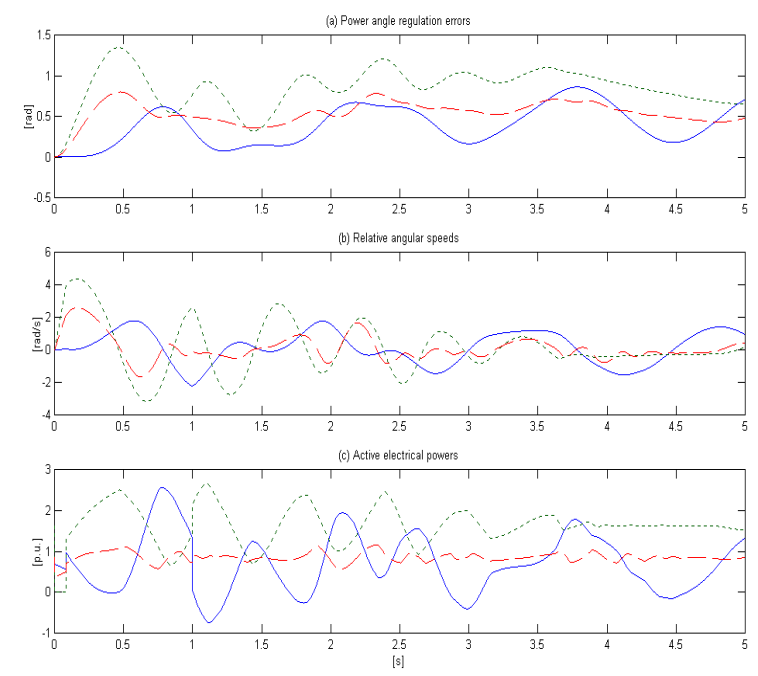

Fig. 3. Robust adaptive control in [15] [Generator 1 (solid), Generator 2 (dot), Generator 3 (dash)]: a) Power angle regulation errors $\delta_{i}-\delta_{i s}$ $(1 \leq i \leq 3)$; b) Relative angular speeds $\omega_{i}(1 \leq i \leq 3)$; c) Active electrical powers $P_{e i}(1 \leq i \leq 3)$.

[4] Y. Chen, C. Zhang, Z. Hu and X. Wang, "A new approach to real time measurement of power angles of generators at different locations for stability control", Power Engineering Society Winter Meeting, vol. 2, pp. 1237-1242, 2000.

[5] G. Damm, R. Marino and F. Lamnabhi-Lagarrigue, "Adaptive nonlinear excitation control of synchronous generators", in A. Zinober, D. Owens (eds), Nonlinear and Adaptive Control, Berlin, Springer Verlag, pp. 6576, 2003.

[6] G. Damm, R. Marino and F. Lamnabhi-Lagarrigue, "Adaptive nonlinear output feedback for transient stabilization and voltage regulation of power generators with unknown parameters", International Journal of Robust and Nonlinear Control, vol. 14, pp. 833-855, 2004.

[7] G. Damm, R. Marino, F. Lamnabhi-Lagarrigue and C.M. Verrelli, "Transient stabilization and voltage regulation of a synchronous generator", in F. Lamnabhi-Lagarrigue, S. Laghrouche, A. Loria, E. Panteley (eds), Taming Heterogeneity and Complexity of Embedded Control, London, ISTE, pp. 211-225, 2006.

[8] F.P. de Mello, "Measurement of synchronous machine rotor angle from analysis of zero sequence harmonic components of machine terminal voltage, IEEE Transactions on Power Delivery, vol. 9, pp. 1770-1777, 1994.

[9] Y. Guo, D.J. Hill and Y. Wang, "Nonlinear decentralized control of large-scale power systems", Automatica, vol. 36, pp. 1275-1289, 2000.

[10] Y. Guo, D.J. Hill and Y. Wang, "Global transient stability and voltage regulation for power systems", IEEE Transactions on Power Systems, vol. 16 , pp. $678-688,2001$.

[11] Q. Lu, S. Mei, W. Hu, F.F. Wu, Y. Ni and T. Shen, "Nonlinear decentralized disturbance attenuation excitation control via new recursive design for multi-machine power systems", IEEE Transactions on Power Systems, vol. 16, pp. 729-736, 2001.

[12] Q. Lu, Y.Z. Sun and S. Mei, Nonlinear control systems and power system dynamics, Boston, Kluwer, 2000.

[13] J. Machowski, S. Robak, J.W. Bialek, J.R. Bumby and N. Abi-Samra, "Decentralized stability-enhancing control of synchronous generator", IEEE Transactions on Power Systems, vol. 15, pp. 1336-1344, 2000.

[14] R. Marino, G. Damm and F. Lamnabhi-Lagarrigue, "Adaptive nonlinear excitation control of synchronous generators with unknown mechanical power", in A. Isidori, F. Lamnabhi-Lagarrigue, W. Respondek (eds), Nonlinear Control in the Year 2000, London, Springer Verlag, pp. 107-121, 2001.

[15] R. Marino, T. Shen and C.M. Verrelli, "Robust adaptive transient stabilization of a synchronous generator with parameter uncertainty", European Journal of Control, vol. 12, pp. 135-148, 2006.

[16] R. Marino and P. Tomei, "Robust adaptive state-feedback tracking control for nonlinear systems", IEEE Transactions on Automatic Control, vol. 43, pp. 84-89, 1998.

[17] R. Marino and P. Tomei, "Nonlinear output feedback tracking with almost disturbance decoupling", IEEE Transactions on Automatic Control, vol. 44, pp. 18-28, 1999.

[18] R. Ortega, M. Galaz, A. Astolfi, Y. Sun and T. Shen, "Transient stabilization of multimachine power systems with nontrivial transfer conductances", IEEE Transactions on Automatic Control, vol. 50, pp. 60-75, 2005.

[19] P.W. Sauer and M.A. Pai, Power system dynamics and stability, Upper Saddle River, Prentice Hall, 1998.

[20] T. Shen, S. Mei, Q. Lu, W. Hu and K. Tamura, "Adaptive nonlinear excitation control with $\mathcal{L}_{2}$ disturbance attenuation for power systems", Automatica, vol. 39, pp. 81-89, 2003.

[21] T. Shen and K. Tamura, "Robust $\mathcal{H}_{\infty}$ control of uncertain nonlinear systems via state feedback", IEEE Transactions on Automatic Control, vol. 40, pp. 766-768, 1995.

[22] Y. Tan and Y. Wang, "Augmentation of transient stability using a superconducting coil and adaptive nonlinear control", IEEE Transactions on Power Systems, vol. 13, pp. 361-366, 1998.

[23] Y. Wang, D. Cheng, C. Li and Y. Ge, "Dissipative Hamiltonian realization and energy-based $\mathcal{L}_{2}$-disturbance attenuation control of multimachine power systems", IEEE Transactions on Automatic Control, vol. 48, pp. 1428-1433, 2003.

[24] Y. Wang, D. Cheng, Y. Liu and C. Li, "Adaptive $\mathcal{H}_{\infty}$ excitation control of multimachine power systems via the Hamiltonian function method", International Journal of Control, vol. 77, pp. 336-350, 2004.

[25] Y. Wang, G. Guo and D.J. Hill, "Robust decentralized nonlinear controller design for multimachine power systems", Automatica, vol. 33, pp. $1725-1733,1997$.

[26] Y. Wang and D.J. Hill, "Robust nonlinear coordinated control of power systems", Automatica, vol. 32, pp. 611-618, 1996.

[27] Y. Wang, D.J. Hill, R.H. Middleton and L. Gao, "Transient stabilization of power systems with an adaptive control law", Automatica, vol. 30, pp. 1409-1413, 1994.

[28] Z. Xi, D. Cheng, Q. Lu and S. Mei, "Nonlinear decentralized controller design for multimachine power systems using Hamiltonian function method", Automatica, vol. 38, pp. 527-534, 2002.

[29] Z. Xi, G. Feng, D. Cheng and Q. Lu, "Nonlinear decentralized saturated controller design for power systems", IEEE Transactions on Control Systems Technology, vol. 11, pp. 539-547, 2003.

[30] A. I. Zecevic, G. Neskovic and D.D. Siljak, "Robust decentralized exciter control with linear feedback", IEEE Transactions on Power Systems, vol. 19, pp. 1096-1103, 2004. 